\title{
Magnetic Properties of Silicate Glasses Dispersing Iron Oxide Clusters
}

\author{
Kazuhiro NAGATA, Miyuki HAYASHI and Masahiro SUSA
}

\author{
Department of Metallurgical Engineering, Tokyo Institute of Technology \\ Ookayama, Meguro-ku, Tokyo 152, Japan
}

\begin{abstract}
The as-quenched silicate glasses containing iron oxides obey the magnetic structure model that both magnetic clusters and free iron ions in the glass matrix bear the magnetism. The annealing of $20 \mathrm{CaO} \cdot 20 \mathrm{SiO}_{2} \cdot 7 \mathrm{Fe}_{2} \mathrm{O}_{3} \cdot 6 \mathrm{FeO}$ glasses at $973 \mathrm{~K}$ in vacuum produced iron oxide particles with the diameter of 2.5-11.5nm. The phase was identified to be $\mathrm{Fe}^{3+}\left(\mathrm{Fe}^{3+}{ }_{1.30} \mathrm{Fe}^{2+}{ }_{0.55} \mathrm{~V}_{0.15}\right) \mathrm{O}_{4}$ in the inverse spinel structure. The growth of particles proceeded predominantly by diffusion of $\mathrm{Fe}^{3+}$ ions. The particles exhibited superparamagnetism and the effective anisotropy energy constant was inversely proportional to the particle diameter. The thermoremanent magnetization measurements show non-interacting particles as explained by Neel's theory. On the other hand, ac susceptibility measurements show interacting particles as interpreted by Dormann's model. The results indicate the different sensitivity of the above two types of measurements on the superparamagnetic relaxation of particles.
\end{abstract}

\section{I . INTRODUCTION}

The glasses having magnetic clusters can be prepared by annealing silicate glasses containing iron oxides below the glass transition temperature. During annealing process most of iron ions form iron oxide particles, whereas the rest of iron ions remain in the glass matrix. It has been known only that clusters precipitated in the glass matrix by annealing are made of $\mathrm{Fe}_{3} \mathrm{O}_{4}$ or $\gamma-\mathrm{Fe}_{2} \mathrm{O}_{3}$ [1-2]. These particles have magnetic moments and show superparamagnetism above the blocking temperature. An application of the silicate glasses containing magnetic particles could be to magnetooptical devices and requires the investigation of the growth process of particles and the magnetic properties such as the magnetic interaction between clusters. The magnetic interaction between particles can be realized by the comparison of the magnitude of the dipole interaction between particles with that of the anisotropy energy. Particles distributed at random with dipole interactions more predominant than the anisotropy energy would exhibit spin-glass-ordering of the magnetic moments at low temperatures. The blocking is not due to superparamagnetic relaxation of non-interacting particles, but due to ordering of interacting magnetic moments. On the other hand, the magnetic moment of particles with negligibly weak interactions fluctuates around the energy minimum corresponding to each easy axis below the blocking temperature. The relaxation of the magnetic moments will occur by the transition over the energy barrier due to the anisotropy energy above the blocking temperature. Jonson et al. [3] have measured the ac susceptibility on frozen ferrofluids containing single domain particles of $\mathrm{Fe}_{3} \mathrm{O}_{4}$ and showed that the cusp in the susceptibility shifts to higher temperatures as the particle concentration increases. This indicates that the dipole interactions between particles affect the energy barrier for thermal relaxation of the magnetic moment of a particle, which is attributed to the anisotropy energy.

\section{EXPERIMENTALS}

A master glass of 40 (mole $\%) \mathrm{CaO}-40 \mathrm{SiO}_{2}-20$ $\mathrm{Fe}_{2} \mathrm{O}_{3}$ was prepared from dried and reagent grade $\mathrm{SiO}_{2}, \mathrm{Fe}_{2} \mathrm{O}_{3}$ and $\mathrm{CaO}$ powders. A weighed mixture of the reagent oxides was melted in a platinum crucible at $1723 \mathrm{~K}$ for $3.6 \mathrm{ks}$ in air, poured onto a water-cooled copper plate and then crushed. About $250 \mathrm{mg}$ of glasses were melted again in a platinum crucible of $5 \mathrm{~mm}$ in outer diameter, $0.1 \mathrm{~mm}$ in wall thickness and $10 \mathrm{~mm}$ in height at $1723 \mathrm{~K}$ in a flow of $\mathrm{CO}-\mathrm{CO}_{2}$ mixture, $\mathrm{CO}_{2}, \mathrm{O}_{2}$ or air. After being held more than $21.6 \mathrm{ks}$, the glasses were quenched into water. Subsequently the glass samples were annealed in evacuated quartz tubes to produce iron oxide paticles at $973 \mathrm{~K}$ for 0.3 to $86.4 \mathrm{ks}$.

The amorphous state of glasses was examined by $\mathrm{X}$-ray diffraction. The spacial distribution and size of iron oxide clusters and particles were determined from photographs by a transmission microscopy (TEM). The magnetic hyperfine field, the magnitude of magnetic moments and the number density of particles in glass samples were determined by Mossbauer spectroscopy. The magnetization was measured by a vibrating sample magnetometer (VSM). The dc magnetization and ac susceptibility of samples were measured by a 
superconducting quantum interference device (SQUID) magnetometer (Quantum Design). Dc magnetization measurements were carried out at $300 \mathrm{~K}$ with increasing magnetic field from 0 to $10 \mathrm{kOe}$. On zero-field-cooled (ZFC) and field-cooled (FC) samples, dc magnetization measurements were carried out under a static dc field of 5000 e with increasing temperature from 2 to $200 \mathrm{~K}$. Ac susceptibilities were measured on a ZFC sample in frequency range of $0.03-3.62 \mathrm{~Hz}$ with increasing temperature from 8 to $15 \mathrm{~K}$. The amplitude of ac magnetic field width was fixed at $2 \mathrm{Oe}$.

\section{RESULTS AND DISCUSSIONS}

\section{A. As-quenched silicate glasses}

TEM view for the sample in which the ratio $(x)$ of number of $\mathrm{Fe}^{3+}$ ion to the total iron ion was 0.73 showed light and shade. Then, iron ions seem to be distributed inhomogeneously. It can be considered that this sample consists of two phases, that is, microcrystallites called clusters and the glass matrix containing iron ions. Both for the samples having smaller values of $\mathrm{x}$ and for those having larger $x$ than 0.67 , the number of clusters became smaller.

As shown in Figure 1, the temperature dependences of the ZFC and FC static magnetizations for this sample shows three noticeable features: (1) the ZFC magnetization shows a characteristic cusp at $10 \mathrm{~K}$, (2) the FC magnetization is a little larger than the ZFC magnetization at temperatures between 10 and $170 \mathrm{~K}$, and (3) the ZFC and FC magnetizations meet at $170 \mathrm{~K}$. The cusp is due to spin-glass-like behavior of iron ions in glass matrix.

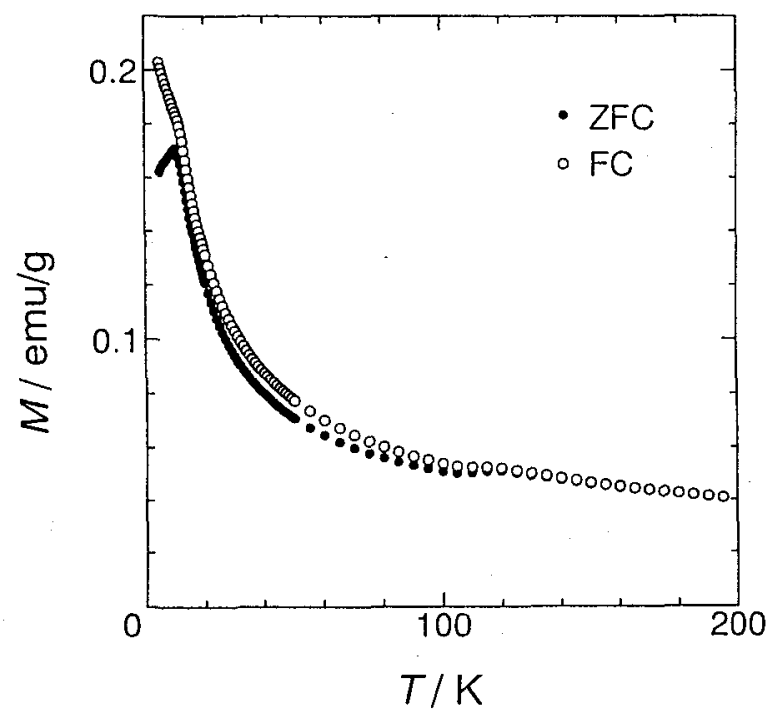

Fig.1 Temperature dependence of ZFC and FC static magnetizations
Namely, if iron ions have spin-glass ordering at below $10 \mathrm{~K}$, the magnetic moments of iron ions are frozen in random directions. With increasing temperature, the magnetic moments are directed more easily along external magnetic field by virtue of thermal energy, resulting larger magnetization. However, as the temperature is even higher, thermal fluctuation of the magnetic moments gets stronger, and the magnetization becomes smaller. Moreover, since the FC magnetization below $10 \mathrm{~K}$ decreases with increasing temperature, some iron ions in the glass matrix have paramagnetism. From the feature (2), the magnetic moments of clusters for the ZFC samples are directed easy axes attributed to the magnetic anisotropy, and thus are fixed at random directions at lower temperature, while those for the FC samples are directed along the magnetic field. Therefore, the FC magnetization is a little larger than the ZFC magnetization. Moreover, the feature (3) means that all clusters are in a superparamagnetic state above $170 \mathrm{~K}$.

\section{B. Silicate glasses precipitating iron oxide particles}

The X-ray diffraction profiles for the samples annealed at $973 \mathrm{~K}$ showed that the fine particles of $\gamma-\mathrm{Fe}_{2} \mathrm{O}_{3}, \mathrm{Fe}_{3} \mathrm{O}_{4}$ or a solid solution of them was precipitated in the glass matrix. The Mossbauer spectra at $77 \mathrm{~K}$ for annealed samples were deconvoluted into hyperfine splitting due to the particles and quadrapole splitting due to free $\mathrm{Fe}^{2+}$ and $\mathrm{Fe}^{3+}$ ions in the matrix. The phase can be expressed as the following formula; $\mathrm{Fe}^{3+}\left(\mathrm{Fe}^{3+}{ }_{(5-2 \mathrm{y}) / 3}\right.$ $\mathrm{Fe}^{2+} \mathrm{V}_{(1-\mathrm{y}) / 3} \mathrm{O}_{4} \cdot \mathrm{y}$ are 0.57 and 0.42 for annealing time of 0.3 and $86.4 \mathrm{ks}$, respectively. The size distribution of particles obtained from TEM view gives its average diameter of $6.47 \pm 1.77 \mathrm{~nm}$.

The magnetic hyperfine field for glass sample annealed was calculated from the Mossbauer spectrum and decreased with increasing temperature. According to a model proposed by Morup [4], the magnetic moment of a particle fluctuates thermally and the thermal average for the magnetic moments of particles gives the magnetic hyperfine field as a function of temperature. The magnetic hyperfine field can be expressed by the equation; $\mathrm{H}_{\mathrm{obs}}=\mathrm{H}_{0}(1-\mathrm{kT} / \kappa \mathrm{v})$ for $\mathrm{kT} / \kappa \mathrm{v}<1$, where $\kappa$ is the effective anisotropy energy constant and $\mathrm{v}$ is the particle volume. $\kappa$ is proportional to the reciprocal of average particle diameter, which is the ratio of surface area to volume for a particle. This means that $\kappa$ near the surface of particles is larger than that inside the particles. The measured magnetization of glass having particles 
is smaller by a factor of $1 / 4$ than the calculated one. This discrepancy can be explained by the pinning of spins near the particle surface due to the large anisotropy constant.

For a single domain particle having uniaxial anisotropy, the superparamagnetic relaxation time $\tau$ is expressed by $\tau=\tau_{0} \exp (\mathrm{E} / \mathrm{kT})$, where $\mathrm{E}$ is the activation energy for the reversal of the magnetic moment of a particle between two easy directions. Accordingly, the magnetic state of particles depends upon the measurement time $\tau_{m}$ which is required for observation. If $\tau<\tau_{\mathrm{m}}$ for the reversal of the magnetic moment, it is observed that the particles are in the superparamagnetic state. In contrast, if $\tau>\tau_{m}$, the particles is in the blocked state. At the average blocking temperature $T_{B}, \tau$ $=\tau_{\mathrm{m}}$. The remanent magnetization gradually decreased with increasing temperature. This means that larger particles are still in the blocked state even at higher temperatures, because the particles have the size distribution. According to Neel's theory for non-interacting particles, $\mathrm{E}=\kappa \mathrm{v}$, where $v=\pi d^{3} / 6$. The magnitude of $\tau_{0}$ is in the reasonable range of $10^{-9}-10^{-11} \mathrm{~s}$ and a value of $\kappa$ of $4.0 \times 10^{4} \mathrm{~J} / \mathrm{m}^{3}$ is in fair agreement with $5 \times 10^{4} \mathrm{~J} / \mathrm{m}^{3}$ obtained from the low-temperature Mossbauer spectra. These indicate that there is no magnetic interaction between particles. On the other hand, the average blocking temperature $T_{B}$ can be defined as the temperature at which the remanent magnetization is the half of that at oK. Figure 2 shows the relation between $\log _{10} \tau_{m}$ and $1 / T_{B}$ according to Neel's theory.

In ac susceptibility measurements, the average blocking temperature can be defined as the

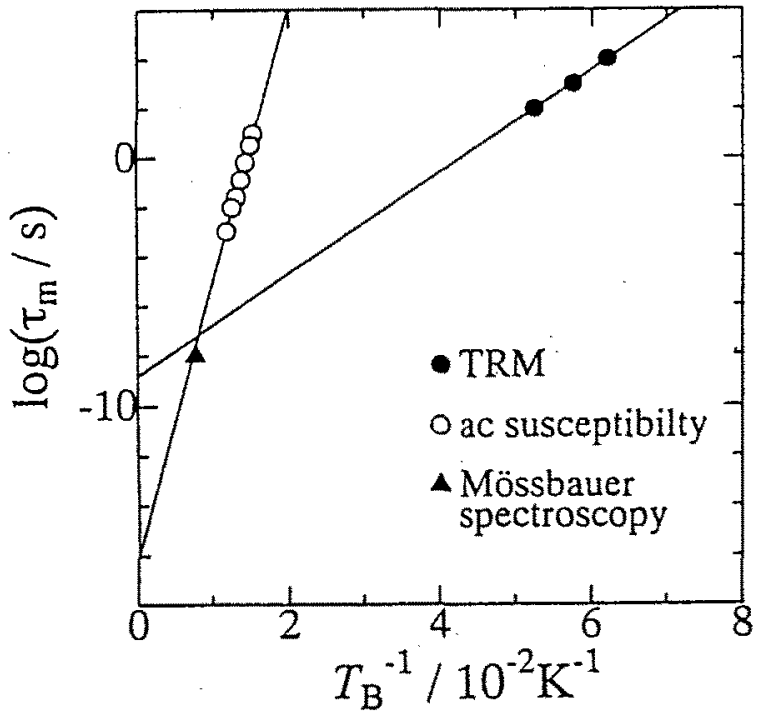

Fig.2 The relation between $\log _{10} \tau_{m}$ and $1 / \mathrm{T}_{B}$ temperature at which the maxima of real parts of susceptibilities appear. From Figure $2, \tau_{0}=10^{-16} \mathrm{~s}$ and $\kappa=20.6 \times 10^{4} \mathrm{~J} / \mathrm{m}^{3}$. However, these values are not reasonable. This indicates that the interaction between particles can not be neglected. According to Dorman's model [5] for the superparamagnetic relaxation of interacting particles with uniaxial anisotropy, the activation energy can be expressed by $\mathrm{E}=\kappa \mathrm{v}+\mathrm{E}_{\text {int }}$, where $\mathrm{E}_{\text {int }}$ is the activation energy due to the interaction between particles. By this model, $\tau_{0}=10^{-10.8} \mathrm{~s}$ and $\mathrm{E}_{\mathrm{int}} / \mathrm{k}=170 \mathrm{~K}$. In Mossbauer spectroscopy measurement, the average blocking temperature can be defined as the temperature at which the ratio of the area for hyperfine splitting to the whole spectral area is half of that at $0 \mathrm{~K}$. $T_{B}=133 \mathrm{~K}$ for the measurement time of about $10^{-8} \mathrm{~s}$, as shown in Figure 2.

\section{CONCLUSIONS}

The as-quenched silicate glasses containing iron oxides obey the magnetic structure model that both magnetic clusters and free iron ions in the glass matrix bear magnetism. The magnetic clusters can be more easily precipitated in the sample having the value $x$ close to that of $\mathrm{Fe}_{3} \mathrm{O}_{4}(\mathrm{x}=0.67)$. The annealed silicate glasses precipitate iron oxide particles in the narrow range $2.5-11.5 \mathrm{~nm}$. The effective anisotropy energy constant near the surface of particles is larger than that inside the particles. The thermoremanent magnetization measurements show noninteracting particles as explained by Neel's theory. On the other hand, ac susceptibility measurements show interacting particles as interpreted by Dormann's model.

\section{REFERENCES}

[1]M.Hayashi, M.Susa, S.Noda and K.Nagata, J. Appl.Phys.,vol.80[12]p.6906-6910,1996.

[2]M.Hayashi, M.Susa, T.Maruyama and K.Nagata, J.ElectronicMaterials, Vol24[8],p.983$989,1995$.

[3]T.Jonsson, P.Svedlindh and P.Nordblad, J. Magn. Magn.Mater.,vol.140-144,p401,1995.

[4]S.Morup,J.Magn.Magn.Mater.,vol.37,p.39,1983.

[5]J.L.Dormann, L.Besais and D.Fiorani, J.Phys.C: Solid State Phys., vol.21,pp.2015,1988. 